\title{
Combining Cell-Based Therapies and Neural Prostheses to Promote Neural Survival
}

\author{
Andrew K. Wise • James B. Fallon • Alison J. Neil • \\ Lisa N. Pettingill • Marilyn S. Geaney • \\ Stephen J. Skinner • Robert K. Shepherd
}

Published online: 9 September 2011

(C) The American Society for Experimental NeuroTherapeutics, Inc. 2011

\begin{abstract}
Cochlear implants provide partial restoration of hearing for profoundly deaf patients by electrically stimulating spiral ganglion neurons (SGNs); however, these neurons gradually degenerate following the onset of deafness. Although the exogenous application of neurotrophins (NTs) can prevent SGN loss, current techniques to administer NTs for long periods of time have limited clinical applicability. We have used encapsulated choroid plexus cells (NTCells; Living Cell Technologies, Auckland, New Zealand) to provide NTs in a clinically viable manner that can be combined with a cochlear implant. Neonatal cats were deafened and unilaterally implanted with NTCells and a cochlear implant. Animals received chronic electrical stimulation (ES) alone, NTs alone, or combined NTs and ES (ES + NT) for a period of as much as 8 months. The opposite ear served as a deafened unimplanted control. Chronic ES alone did not result in increased survival of SGNs or their peripheral processes. NT treatment alone resulted in greater SGN survival restricted to the upper basal cochlear region and an increased density of SGN peripheral processes. Importantly,
\end{abstract}

Electronic supplementary material The online version of this article (doi:10.1007/s13311-011-0070-0) contains supplementary material, which is available to authorized users.

A. K. Wise $(\bowtie) \cdot$ J. B. Fallon · A. J. Neil • L. N. Pettingill •

R. K. Shepherd

The Bionics Institute,

384-388 Albert Street,

East Melbourne 3002, Australia

e-mail: awise@bionicsinstitute.org

\section{A. K. Wise $\cdot$ J. B. Fallon $\cdot$ R. K. Shepherd}

The Department of Otolaryngology, University of Melbourne,

Victoria 3002, Australia

M. S. Geaney $\cdot$ S. J. Skinner

Living Cell Technologies Limited,

Manukau 2025, New Zealand chronic ES in combination with NTs provided significant SGN survival throughout a wider extent of the cochlea, in addition to an increased peripheral process density. Re-sprouting peripheral processes were observed in the scala media and scala tympani, raising the possibility of direct contact between peripheral processes and a cochlear implant electrode array. We conclude that cell-based therapy is clinically viable and effective in promoting SGN survival for extended durations of cochlear implant use. These findings have important implications for the safe delivery of therapeutic drugs to the cochlea.

Keywords Cell-based therapy - Cochlear implantation . Neurotrophin $\cdot$ Nerve protection $\cdot$ Electrical stimulation

\section{Introduction}

In 2005, the World Health Organization estimated that 278 million people were living with a significant hearing impairment. The most common form of impairment is a sensorineural hearing loss (SNHL), which occurs when the sensory hair cells of the cochlea are damaged or absent. For patients with a severely profound SNHL, the only clinical treatment is a cochlear implant, which is a neural prosthesis designed to electrically stimulate spiral ganglion neurons (SGNs) to partially restore hearing to deaf patients. However, SNHL also results in the gradual degeneration of SGNs, the target cells of the cochlear implant [1-3]. SGN degeneration is characterized by retraction of the peripheral processes of the SGNs and ultimately leads to degeneration of the SGN soma and central process $[4,5]$. It is believed that a loss of endogenous neurotrophic support normally provided by the sensory hair cells $[6,7]$ is the primary cause of SGN death, a factor likely to limit the effectiveness of cochlear implants. Therefore, research focusing on strategies to prevent loss of the SGNs and 
their peripheral processes following deafness should lead to improvements in cochlear implant performance [8].

Administration of neuroprotective agents (e.g., the neurotrophins [NTs], brain derived neurotrophic factor, and NT3) in profoundly deafened cochleae has been shown to prevent SGN loss [9-16]. However, the survival promoting effects were short lived once the NT delivery had ceased [17, 18], suggesting that ongoing delivery is required for lasting effects. Combining NT delivery with chronic intracochlear electrical stimulation (ES) from a cochlear implant can enhance SGN survival [8], even after the withdrawal of the exogenous NT supply [18], indicating the potential synergies between treatment with NTs and chronic ES.

In most studies to date, mini-osmotic pumps have been used to deliver NTs over relatively short time periods (weeks) to the cochleae of deaf rodents $[8,9,11-15,17,18]$. The use of mini-osmotic pumps in rodent deafness models, in which SGNs degenerate much more rapidly than in other species, such as the cat (in months [4]) and human (in years [19]), has limited the examination of NT therapies to relatively short timeframes. Furthermore, the limited reservoir and significant risk of infection associated with mini-pumps reduces their potential clinical application [20]. Consequently, the promise of NT therapy to rescue SGNs after SNHL has yet to be realized, and effective and safe drug administration strategies for application in the inner ear remains elusive. The use of cell-based therapies provides a clinically viable NT delivery technique that is less susceptible to adverse side effects than pump-based delivery methods [21-23]. In the present study, choroid plexus cells (NTCells, Living Cell Technologies, Auckland, New Zealand), which produce and release a cocktail of NTs [24], were encapsulated in biocompatible alginate and implanted into the neonataldeafened cat cochlea in combination with a cochlear implant electrode array. The results showed that treatment with cellbased NT therapy for an extended duration (as much as 8 months) provided protection of SGNs and their peripheral processes from deafness-induced changes, and also that survival effects were enhanced by concurrent chronic ES via a cochlear implant.

\section{Methods}

Animals

Twenty-four cats were used in these experiments. All animal procedures were carried out with the approval of the Royal Victorian Eye and Ear Hospital Animal Research and Ethics Committee and conformed to the Code of Practice of the National Health and Medical Research Council of Australia.
Deafening and Implant Surgery

The 4 experimental cohorts are described in Table 1 . Neonatal cats $(n=17)$ were administered a daily subcutaneous injection of the aminoglycoside neomycin sulfate $(60 \mathrm{mg} / \mathrm{kg}$ s.c.) until they were profoundly deaf (approximately 3 weeks $[25,26])$. Deafness was assessed by the absence of an auditory brainstem response (ABR) to an acoustic click recorded using standard electrophysiological techniques [27]. Briefly, animals were anesthetized with xylazine (4 mg/kg s.c.) and ketamine $(20 \mathrm{mg} / \mathrm{kg}$ i.m.) and placed on a heating pad $\left(37^{\circ} \mathrm{C} \pm 1^{\circ} \mathrm{C}\right)$ in a sound attenuated room. ABRs were recorded differentially using subcutaneous stainless steel electrodes (vertex positive, neck negative, and thorax ground) in response to $100 \mu$ s clicks at $98 \mathrm{~dB}$ peak equivalent sound pressure level. Signals were amplified by a factor of $10^{5}$ and were bandpass filtered (150 Hz to $3 \mathrm{kHz}$ ). Only animals treated with neomycin that exhibited a hearing loss in excess of $>50 \mathrm{~dB}$ to a clickevoked ABR were used in the present study.

At 2 months of age, deafened animals underwent cochlear implant surgery as previously described [27]. Briefly, the cochlea was exposed using aseptic surgical techniques and a small incision was made in the round window. Alginate capsules (average, $n=23 \pm 1.2$ SEM) containing NTCells (Living Cell Technologies) (NT and $\mathrm{ES}+\mathrm{NT}$ group) or empty capsules (ES group) were delivered via a cannula into the scala tympani and the intracochlear electrode array was subsequently inserted approximately $8 \mathrm{~mm}$ into the scala tympani (Fig. 1). The electrode lead wire was secured and exited the skin via a percutaneous opening below the nape of the neck.

\section{NTCells}

Cells were harvested by Living Cell Technologies from the choroid plexus of neonatal pigs, as previously described [28]. The pigs $(2-6 \mathrm{~kg})$ were sourced from a pathogen-free and isolated colony on the Auckland Islands (New Zealand). The choroid plexus was removed, finely dissected, digested with collagenase and allowed to settle before the supernatant was removed and filtered. The preparation

Table 1 Experimental groups, the number of animals used, and the duration of treatment $( \pm$ SEM)

\begin{tabular}{lcc}
\hline Group & Number & Mean duration of treatment \\
\hline ES & 5 & $195 \pm 2.8$ days \\
NT & 6 & $234 \pm 5.3$ days \\
ES + NT & 6 & $217 \pm 8.7$ days \\
Normal & 7 & Age matched \\
\hline
\end{tabular}

$E S$ electrical stimulation; $N T$ neurotrophins 


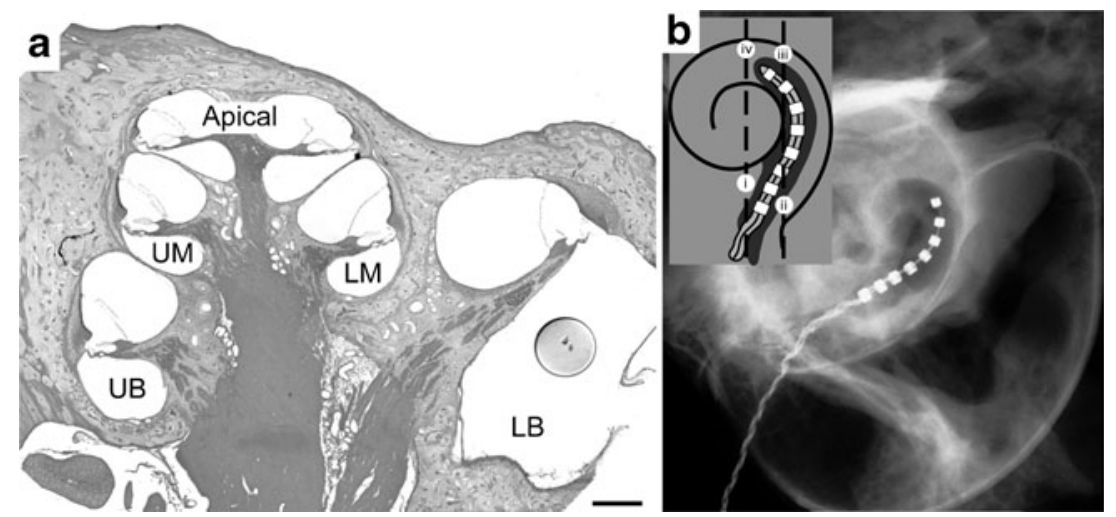

Fig. 1 Low magnification micrograph of the cat cochlea (a) depicting the cochlear regions examined; lower basal (LB), upper basal (UB), lower middle (LM), upper middle (UM), and Apical regions. A capsule containing NTCells is schematically represented in the LB region to provide an approximation of capsule dimensions relative to the scala tympani. Scale bar $=500 \mu \mathrm{m}$. (b) An X-ray of an implanted cochlea showing the electrode array extending into the UB region of the cochlea. The schematic diagram (inset) illustrates the cochlear locations (i-iv) where the tissue response was examined was adjusted to approximately 5000 clusters $/ \mathrm{mL}$ in culture medium before being encapsulated in an alginate membrane using good manufacturing practice techniques previously described [24]. The alginate membrane is biocompatible and semi-permeable, permitting the transfer of secreted NTs, gases, nutrients, and waste products across the membrane and isolating the implanted cells from the immune system of the host [29].

\section{Intracochlear Electrode Array and Chronic Electrical Stimulation}

The intracochlear electrode assembly contained 8 intracochlear platinum electrodes on a silicone carrier and 1 extracochlear platinum ball return electrode. The intracochlear electrodes were $0.3-\mathrm{mm}$ wide with an inter-electrode separation of $0.45 \mathrm{~mm}$. A percutaneous lead wire connected the electrode array to a clinical cochlear implant (CI 24, Cochlear Ltd, Sydney, Australia) and speech processor (ESPrit 3G, Cochlear Ltd, Sydney, Australia) as described previously [26]. Chronic environmentally-derived ES was delivered as monopolar charge-balanced biphasic current pulses ( $8 \mu$ s phase, $25 \mu \mathrm{s}$ gap) at a rate of $400 \mathrm{pps} /$ electrode, consistent with clinical configurations. The ES was provided by clinical speech processors and stimulators, and therefore delivered ES identical to that which a cochlear implant patient would receive in the same sound environment. These sounds included vocalizations of the animals and self-generated sounds (e.g., playing with noisy toys) that were shown to drive the processors and thus deliver ES at perceptible levels. This is 1 of a handful of studies to use such clinical stimulation in an animal model, and the first to combine clinical ES with clinically viable NT delivery. Stimulation commenced 2 weeks after surgery and the speech processors were programmed to map the full dynamic range of the acoustic environment to electrical stimulus levels from $3 \mathrm{~dB}$ below to $6 \mathrm{~dB}$ above the electrically-evoked auditory brainstem response (EABR) thresholds. Standard electrophysiological techniques (as described for ABRs) were used to measure EABRs and thresholds were defined as the current level that elicited a peak-to-trough response amplitude of at least $0.2 \mathrm{mV}$ at a latency of 2 to $3 \mathrm{~ms}$ [26]. Animals received ES in a clinically relevant manner $>16 \mathrm{~h} /$ day, 7 days/week for a period of as much as 8 months. The electrical stimulus did not distress the animals as indicated by an absence of stress behavior, such as withdrawal, aggression, appetite, or weight loss and the continuance of normal behavior, such as hunting and inquisitiveness to environmental stimulants and participation in normal play with other cats. Electrode functioning was assessed by daily monitoring of electrode impedances and monthly measurements of EABR thresholds. Stimulation parameters were adjusted in accordance with any changes observed to EABR thresholds, to ensure that ES was delivered at levels sufficient to activate the auditory pathway.

\section{Histology, Immunohistology, and SGN Quantification}

At the conclusion of the treatment period, the animal was euthanized and intracardially perfused with $4 \%$ paraformaldehyde. The cochleae were removed, locally perfused, and postfixed in the same fixative overnight at $4^{\circ} \mathrm{C}$. The cochleae were decalcified in a solution containing $10 \%$ (wt./vol.) ethylenediamine tetra-acetic acid in $0.1 \mathrm{~mol} / \mathrm{L}$ phosphate buffer and then cryopreserved in $30 \%$ sucrose solution. The cochleae were infiltrated with O.C.T. ${ }^{\text {TM }}$ (Tissue-Tek, Torrance, CA) and snap frozen before being sectioned on a cryostat at $12 \mu \mathrm{m}$ and mounted onto Superfrost-Plus slides (Men- 
zel-Gläser, Braunschweig, Germany) [30]. A representative series of sections of the entire cochlea was stained with hematoxylin and eosin.

Immunohistochemistry was performed on cochlear sections to identify the mitochondrial content of the NTCells within the implanted capsules and to analyze the SGN peripheral processes within the osseous spiral lamina (OSL). Standard immunohistochemistry procedures were used, whereby sections were washed and blocked before incubation overnight with the primary antibody. After another wash, sections were incubated in the secondary antibody (Alexa Fluro, Molecular Probes Eugene, OR) and mounted with Vectashield mounting media containing DAPI (Vector Laboratories, CA, USA). Antibodies for cytochrome C oxidase (complex IV, subunit Va; Mitosciences, Eugene, OR) were used on sections containing encapsulated NTCells to detect intracellular mitochondria and obtain evidence of metabolic activity. Sections were imaged on a Zeiss Axioplan microscope (Carl Zeiss, Jena, Germany).

Antibodies to neurofilament-200 (NF-200; Chemicon International, Australia) were used to examine the effects of treatment on the radially projecting peripheral processes of the SGNs. Sections were imaged on a confocal microscope (LSM 510 META, Carl Zeiss, Jena, Germany) and confocal stacks containing up to 10 images were collected. A projected image of the stack was used for analysis. The inbuilt "moment" threshold filter in Image $\mathrm{J}$ software (National Institutes of Health, USA) was used to threshold the image, and a region within the OSL containing the peripheral processes was selected for analysis. The density of NF-200 labelled pixels was determined for each section and averages across the 4 sections $(76-\mu \mathrm{m}$ apart) were calculated for each cochlea.

SGNs were quantified in mid-modiolar sections using a Zeiss microscope by a single observer blinded to the experimental cohorts. SGNs were identified within Rosenthal's canal and counted within the lower basal, upper basal, lower middle, upper middle, and apical cochlear regions (Fig. 1). Only SGNs exhibiting a clear nucleus and nucleoli were counted [27]. The area of Rosenthal's canal was measured using Image $J$ software and the density of the SGNs was determined. SGN density data for each cochlear region were averaged from 5 sections that were spaced $76-\mu \mathrm{m}$ apart, ensuring that no SGN was counted more than once.

\section{Cochlear Response to Implantation}

The tissue response of the cochlea to the presence of the electrode array and the capsules was determined to assess the biocompatibility of the NT therapy. This was done by measuring the percentage of the area of the scala tympani occupied by an inflammatory response (fibrous tissue and new bone). Tissue response was quantified in hematoxylin and eosin-stained sections at 4 cochlear locations within the basal turn (Fig. 1) using a Zeiss Axioplan microscope, which was then analyzed using Image J.

\section{Statistics}

Statistical comparisons of SGN density and peripheral process density were made by comparing the treated cochlea (left) with the unimplanted contralateral control cochlea (right) via a paired $t$ test or a repeated measures (RM) analysis of variance (ANOVA) using $p<0.05$ level of significance. Post hoc comparisons were made using the Holm Sidak method.

\section{Results}

\section{Deafness-Induced Degeneration of SGNs}

The aminoglycoside deafening procedure resulted in bilateral threshold shifts of $>50 \mathrm{~dB}$ to acoustic clicks. Analysis of the cochlear histology indicated that deafening was effective in symmetrically eliminating cochlear hair cells in the organ of Corti (OC). SGN degeneration was characterized by a bilateral reduction in the packing density of the peripheral processes within the OSL and SGN loss within Rosenthal's canal (Fig. 2). Statistical analysis of SGN density indicated that there was significant loss of SGNs in all but the most apical cochlear regions (2-way ANOVA, Holm-Sidak post hoc $p<0.001$ for the lower basal, upper basal, lower middle, and upper middle cochlear regions, and $p>0.05$ for the apical cochlear region) (Fig. 2). As no SGN loss was observed in the apical region, all further analysis of the treatment groups was restricted to the basal and middle cochlear turns.

\section{SGN Survival following ES Treatment}

Deafened cats in the chronic ES group $(n=5)$ received a cochlear implant and empty alginate capsules unilaterally. As previously noted, the systemic application of neomycin resulted in bilateral elimination of the $\mathrm{OC}$ and a secondary reduction in the density of the peripheral processes within the OSL (Fig. 3). There was no significant difference in SGN density within Rosenthal's canal between the ES cochlea and the untreated contralateral cochlea in any cochlear region, indicating that chronic ES did not prevent ongoing SGN loss (RM ANOVA, $p=0.41$ ).

\section{SGN Survival following NT Treatment}

Deafened cats in the NT group $(n=6)$ received a cochlear implant along with encapsulated NTCells 


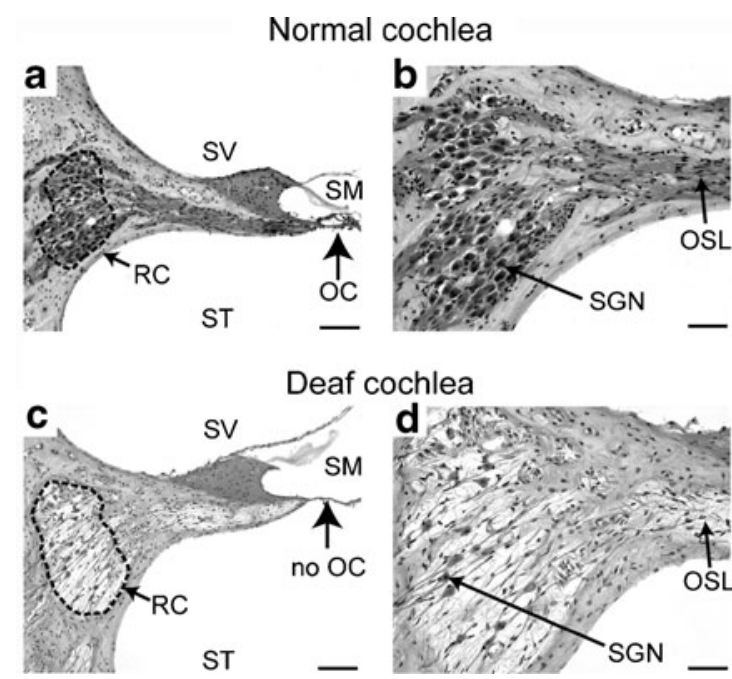

Fig. 2 The upper basal region in mid-modiolar sections from a normal hearing cochlea (a and $\mathbf{b}$ ) and from a cochlea deafened for 8 months (c and $\mathbf{d})$. The normal organ of Corti (OC) (a, arrow) with a full complement of spiral ganglion neurons (SGNs) located within Rosenthal's canal (RC, dotted line) is evident. The deafened cochlea exhibited a total loss of the organ of Corti (c, arrow) with a significant loss of SGNs within Rosenthal's canal and a reduced packing density of peripheral processes within the osseous spiral lamina (OSL) (d).

(Fig. 4) but did not receive chronic ES. Although there was no significant effect of NT treatment on SGN survival throughout the cochlea, further analysis of SGN density in the upper basal region indicated there was a $35 \%$ greater density of SGN in the NT-treated cochlea
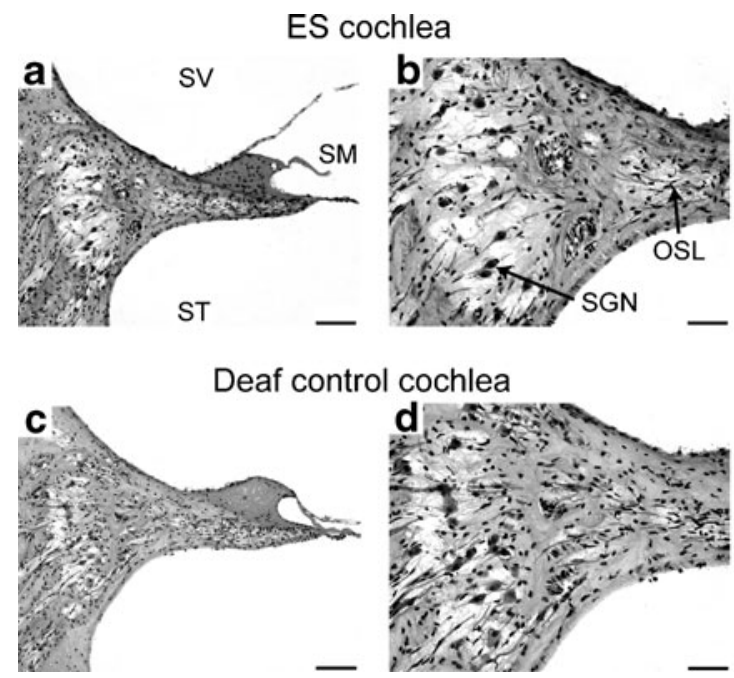

Fig. 3 Mid-modiolar sections of the upper basal cochlear region in an 8 month deafened cochlea that has received chronic ES for six months (a and $\mathbf{b}$ ) and the deafened, untreated contralateral cochlea (c and $\mathbf{d}$ ). The histological images show a complete loss of the organ of Corti in both cochleae as a result of the systemic deafening (a and $\mathbf{c}$ ). There were similar numbers of SGNs in Rosenthal's canal and a thinning of

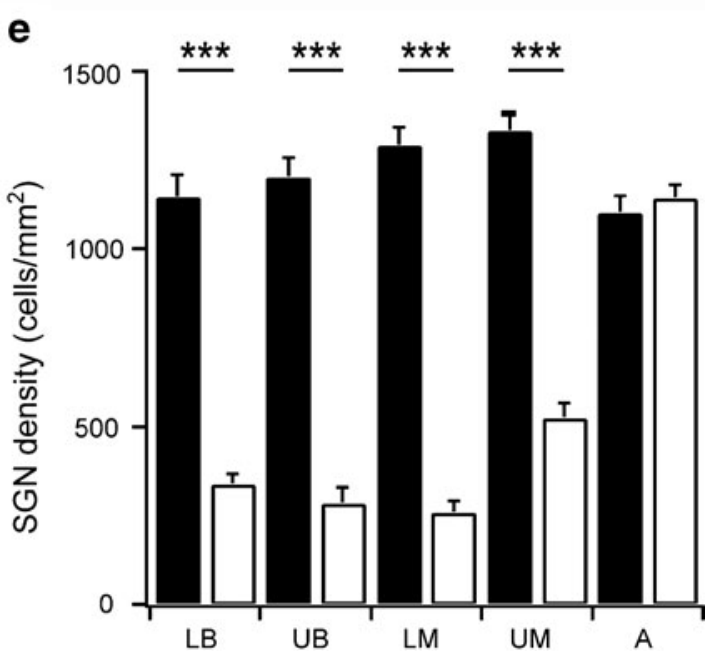

Scale bar $=100 \mu \mathrm{m}$ (a and $\mathbf{c})$ and $50 \mu \mathrm{m}(\mathbf{b}$ and d). Scala media (SM), Scala vestibuli (SV), and scala tympani (ST). (e) Average SGN density ( \pm SEM) within Rosenthal's canal at different cochlear regions in the normal ( $n=12$, black bars) and long-term deaf control $(n=17$, white bars) cochleae. The systemic deafening reduced SGN density in all cochlear regions, except the apical region. $\mathrm{A}=$ apical; $\mathrm{LB}=$ lower basal; $\mathrm{LM}=$ lower middle; $\mathrm{UB}=$ upper basal; $\mathrm{UM}=$ upper middle (see Figure 1 for location of cochlear region). * - significant difference

compared to the right untreated contralateral cochlea, a difference that was statistically significant (paired $t$ test, $p<0.05$ ). These findings indicated that the NT treatment alone provided localized SGN protection to the upper basal region only.

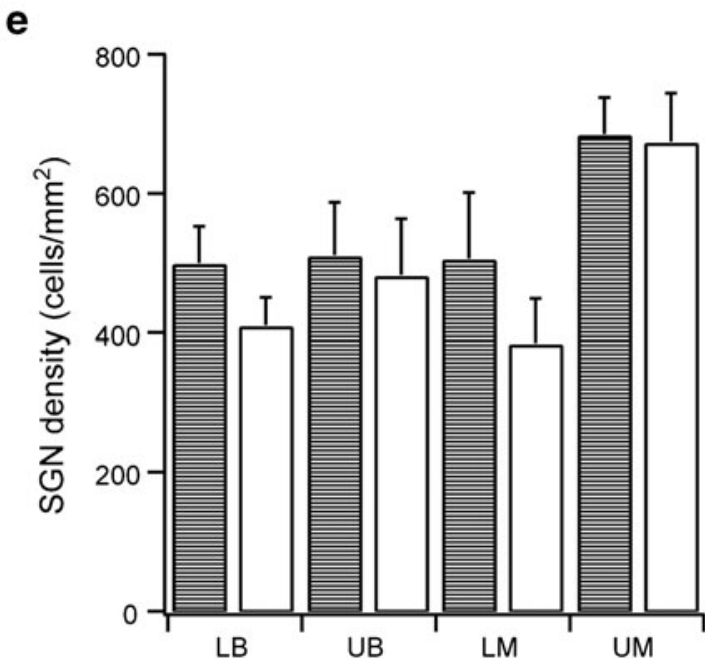

peripheral processes in the OSL (b and d). Scale bar, $100 \mu \mathrm{m}$ (a and c) and $50 \mu \mathrm{m}$ (b and d). (e) Average SGN density ( \pm SEM) within Rosenthal's canal at different cochlear regions in cochleae receiving chronic ES(bars with horizontal lines) and the unimplanted contralateral cochlea (white bars) across all cochlear regions 


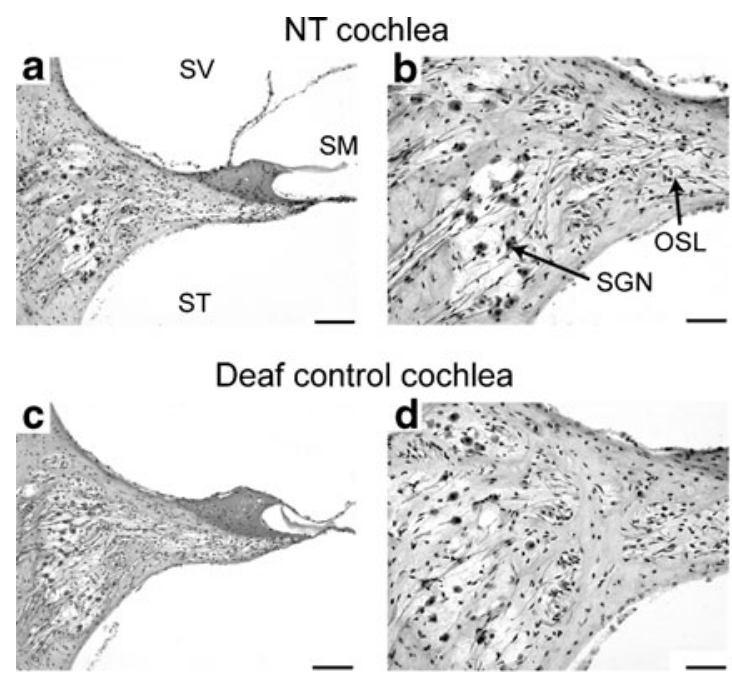

Fig. 4 Mid-modiolar sections of the upper basal cochlear region in a deafened cochlea that had received neurotrophins (NTs) without chronic electrical stimulation (a and b) and the deafened untreated contralateral cochlea (c and $\mathbf{d})$. There was a loss of the organ of Corti in both cochleae (a and $\mathbf{c}$ ) and a small increase in the density of spiral ganglion neurons (SGNs) in the treated cochlea (b) compared to the contralateral cochlea (d). Scale bar $=100 \mu \mathrm{m}(\mathbf{a}$ and $\mathbf{c})$ and $50 \mu \mathrm{m}(\mathbf{b}$ and d). (e) Average SGN density ( \pm SEM) at different cochlear regions

SGN Survival following Combined ES and NT Treatment

Deafened cats in the combined ES + NT group $(n=6)$ received NT treatment in combination with chronic ES. Figure 5 shows examples of mid-modiolar sections from

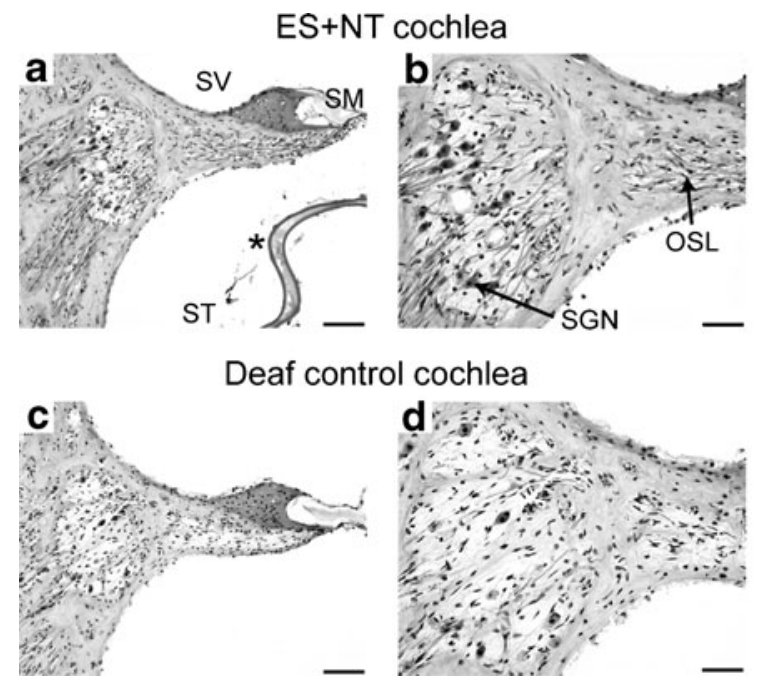

Fig. 5 Mid-modiolar sections of the upper basal cochlear region in an electrical stimulation (ES) + neurotrophins (NTs) treated cochlea (a and $\mathbf{b}$ ) and the unimplanted deaf control cochlea (contralateral side) (c and d). (a) An NTCell capsule is evident $(*)$. There was a greater density of spiral ganglion neurons (SGNs) in the treated cochlea (b) compared to the contralateral control cochlea (d). Scale bar $=100 \mu \mathrm{m}$ (a and $\mathbf{c}$ ) and $50 \mu \mathrm{m}$ (b and d). (e) Average SGN density ( \pm SEM) at different cochlear regions in cochleae receiving ES + NT treatment

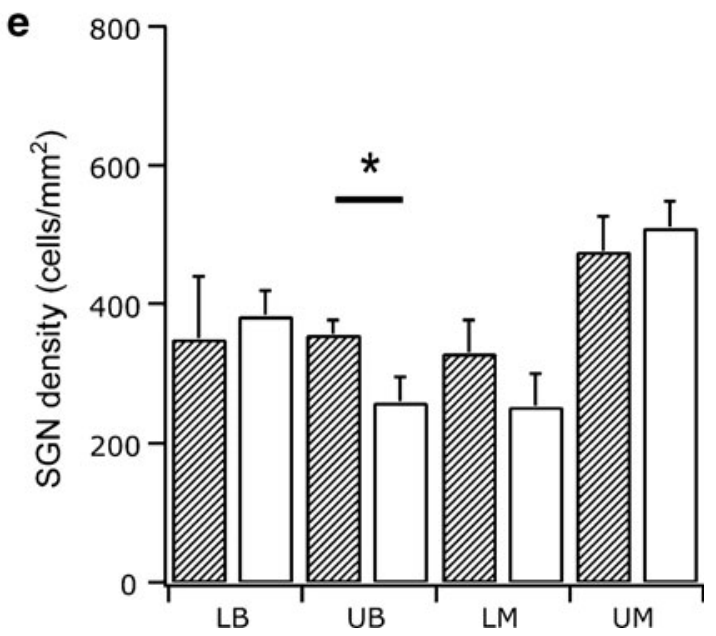

in cochleae receiving NTs (diagonal pattern) and the deafened, untreated contralateral cochlea (white bars). There was a significant difference in the mean SGN density ( $35 \%$ greater) restricted to the upper basal region of cochleae receiving NTs compared to the contralateral cochlea (white bars) (paired $t$ test $[p<0.05]$ ). LB $=$ lower basal; $\mathrm{LM}=$ lower middle; OSL = osseous spiral lamina; $\mathrm{SM}=$ scala media; $\mathrm{SV}=$ scala vestibuli; $\mathrm{ST}=$ scala tympani; $\mathrm{UB}=$ upper basal; $\mathrm{UM}=$ upper middle. ${ }^{*}$ - significant difference

the upper basal turn in an ES + NT treated (Fig. 5a and b) and an untreated control cochlea (Fig. $5 \mathrm{c}$ and d). A capsule containing NTCells is visible in the section (Fig. 5a). There was a significant main effect of ES + NT treatment on the density of SGNs in treated cochleae compared to the

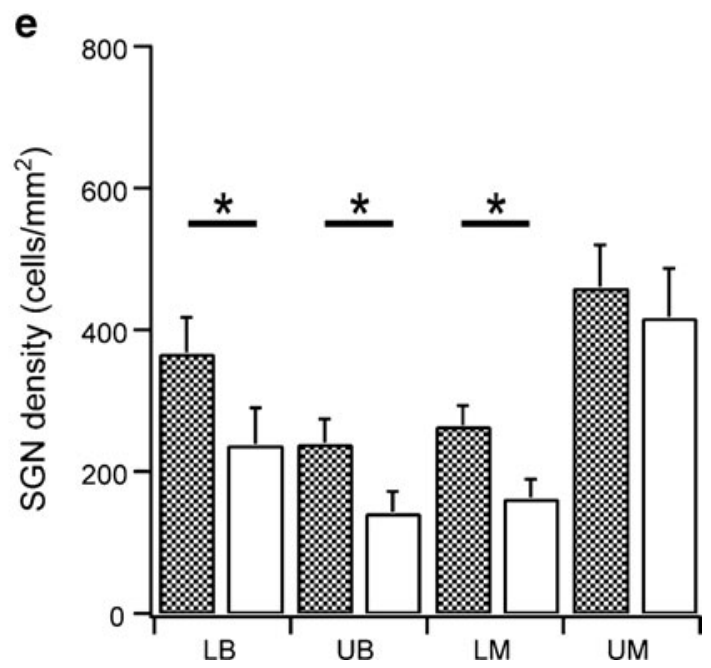

(checked pattern) and the deafened, untreated control cochlea (white bars). The SGN density across all cochlear regions (except the upper middle turn) was greater in the ES + NT treated cochleae compared to the deafened, untreated control cochlea (repeated measures (RM) analysis of variance, $p<0.05)$. $\mathrm{LB}=$ lower basal; $\mathrm{LM}=$ lower middle; OSL $=$ osseous spiral lamina; $\mathrm{SM}=$ scala media; $\mathrm{SV}=$ scala vestibuli; $\mathrm{ST}=$ scala tympani; $\mathrm{UB}=$ upper basal; $\mathrm{UM}=$ upper middle. * - significant difference 
untreated control cochleae (RM ANOVA, $p<0.005$ ). Post hoc analysis indicated that SGN density in the lower basal $(44 \%$ greater SGN density in treated cochlea $[p<$ $0.001])$, upper basal $(68 \%, p<0.02)$, and lower middle regions $(63 \%, p<0.01)$ was significantly greater in ES + NT cochleae compared to the untreated control cochlea. There was no difference in SGN survival in the upper middle turn of the ES + NT cochleae $v s$ their deafened untreated controls. A summary of SGN survival across the different cochlear regions for each treatment group is shown in Table 2.

\section{Peripheral Process Density}

Peripheral processes were immunostained with NF200 and their density measured within the OSL in the basal cochlear region. The stained peripheral processes in OSL cross sections in normal cochleae were typically punctate in appearance (Fig. 6a), indicative of their direct radial projection toward the OC. In contrast, processes in deafened control and treated cochleae were not observed with punctate staining profile, but appeared to have a more disorganized projection profile (Fig. $6 \mathrm{~b}$ and c). Peripheral processes in the normal cochlea occupied on average $16.5 \%$ $( \pm 2.0 \mathrm{SEM})$ of the space within the OSL (Fig. 6d).

The peripheral process density was analyzed for all treatment groups in the basal cochlear region. Examples are shown for peripheral processes in an ES + NT treated cochlea (Fig. 6b) that exhibited a greater density of peripheral processes compared to the untreated control cochlea (Fig. 6c). There was a significant main effect of treatment on the density of peripheral processes (RM ANOVA, $p<0.001)$. Post hoc analysis indicated that there was a significantly greater density of processes in ES + NT treated cochlea (Holm-Sidak, $p<0.001)$ and in NT-treated cochleae $(p<0.001)$ compared to their respective contralateral control cochleae. There was no difference in the density of peripheral processes in the ES-treated cochleae compared to the control. The density of processes in normal cochleae was significantly greater than all treatment groups (1-way ANOVA, $p<0.001$ ).

Table 2 Summary of SGN survival effects for each treatment condition across cochlear region

Lower basal Upper basal Lower middle Upper middle

\begin{tabular}{lllll}
\hline $\mathrm{ES}$ & - & - & - & - \\
$\mathrm{NT}$ & - & $*$ & - & - \\
$\mathrm{ES}+\mathrm{NT}$ & $* * *$ & $* *$ & $* *$ & - \\
\hline
\end{tabular}

$S G N$ spiral ganglion neurons

$*_{p}<0.05 ; * * p<0.02$;

$* * * p<0.001$
Peripheral Process Re-Sprouting

Assessment of the NF200 stained peripheral processes showed re-sprouting processes in the scala tympani and the scala media. Re-sprouting processes were observed in all 3 cohorts, and Fig. 7 depicts examples of re-sprouting processes in cochleae from the ES and NT treatment groups. Processes were observed to project into the cellular epithelial layer on the inner sulcus following their exit from the habenula perforata (Fig. 7a and d) and to project underneath the lip of the inner sulcus near the anchor point of the tectorial membrane (Fig. 7a). Processes were also observed in the scala tympani (Fig. 7a-c) coursing within the fibrous tissue encapsulating the electrode array, as evident by the DAPI-stained fibrotic tissue within the scala tympani. Scala tympani projecting processes were observed to project perpendicular to their normal radial projection and parallel with the intracochlear electrode array (Fig. 7b). Processes were observed to enter the scala tympani through the bony modiolar wall (data not shown) and also underneath the basilar membrane (Fig. 7c). Scala tympani projecting processes were only observed in association with a fibrous tissue reaction to the electrode array.

\section{Tissue Response to Implantation}

The alginate capsules were approximately 600 to $660 \mu \mathrm{m}$ in diameter and contained clusters of NTCells (Fig. 8a). Following implantation for periods of at least 6 months, the capsules were usually detected in the lower and upper basal turns, although some were also occasionally observed in higher turns. There was no evidence of a vigorous tissue response around the capsules, indicating that the alginate capsules were biocompatible. Immunohistological analysis of the NTCells following implantation indicated positive staining for the nuclear marker DAPI, and the mitochondrial marker cytochrome $\mathrm{C}$ oxidase (Fig. 8c), indicating that NTCells were alive and metabolically active at completion of the chronic implantation period.

To quantify the cochlear tissue response to chronic implantation of the electrode array and the alginate capsules (with or without NTCells) the extent of the tissue response within the scala tympani was determined. The tissue response was most prominent in the lower basal region where the electrode array was positioned and most of the capsules were located (Fig. 9a). Some capsules were observed in cochlear regions apical to the electrode array, extending into the upper basal and middle turns (Fig. 9a). The insertion of the electrode array after the implantation of the capsules may have facilitated movement of the capsules apically. In cases where the capsules were observed beyond the electrode array, there was minimal tissue response (irrespective of whether they were empty or contained 


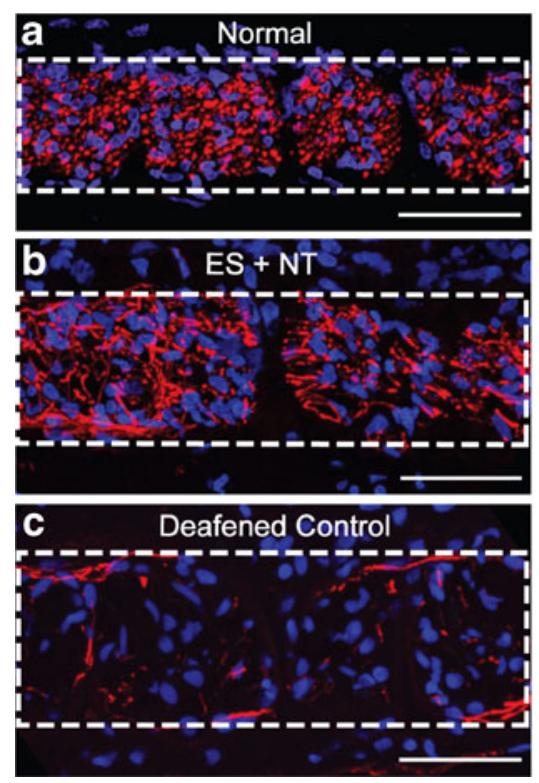

Fig. 6 Confocal images of osseous spiral lamina (OSL) cross sections stained with NF200 (red) and DAPI (blue). The dotted rectangle in (a-c) indicates the OSL in the cochlear region depicted in e (dotted line). (a) Peripheral processes (red) in a normal cochlea were punctate in appearance, indicative of a cross-sectional view of the radially projecting processes, and exhibited a relatively tight packing density within the OSL. The nuclei of a number of glial cells (blue) are also evident within the OSL. (b) Peripheral processes in a cochlea treated with electrical stimulation (ES) + neurotrophins (NTs) project in an ectopic manner, indicated by more laterally projecting processes, but exhibited a greater packing density when compared to the deafened control (contralateral) cochlea shown in (c). Scale bar $=50 \mu \mathrm{m}$. (d)

NTCells). The tissue response was quantified by measuring the area of scala tympani and the area of the tissue response in 4 locations within the lower and upper basal region (Fig. 1b). Analysis of the data indicated that there was no effect of cochlear location (2-way ANOVA, $p=0.19$ ); therefore, the data were collapsed across the 4 cochlear locations. Example micrographs from 2 cochlear locations, 1 and 4, and the average data ( \pm SEM) for the tissue response and for new bone growth is expressed as a percentage of scala tympani cross-sectional area for each cohort (Fig. 9). There was no statistical difference across the experimental cohorts for the tissue response (2-way ANOVA, $p=0.36$ ). Although there was a tendency for a greater amount of new bone growth to occur in location 1 compared to location 4, statistical analysis just failed to reach significance for the main effect of cochlear location (2-way ANOVA, $p=0.05$ ). There was no significant difference in the extent of new bone growth between experimental groups (2-way ANOVA, $p=0.08$ ). Similarly, although there was a trend for the average electrode impedance to increase with time (range, $16-49 \%$ average increase), there was no statistical difference in impedance changes between the experimental cohorts (ANOVA on ranks, $p>0.05$ ).

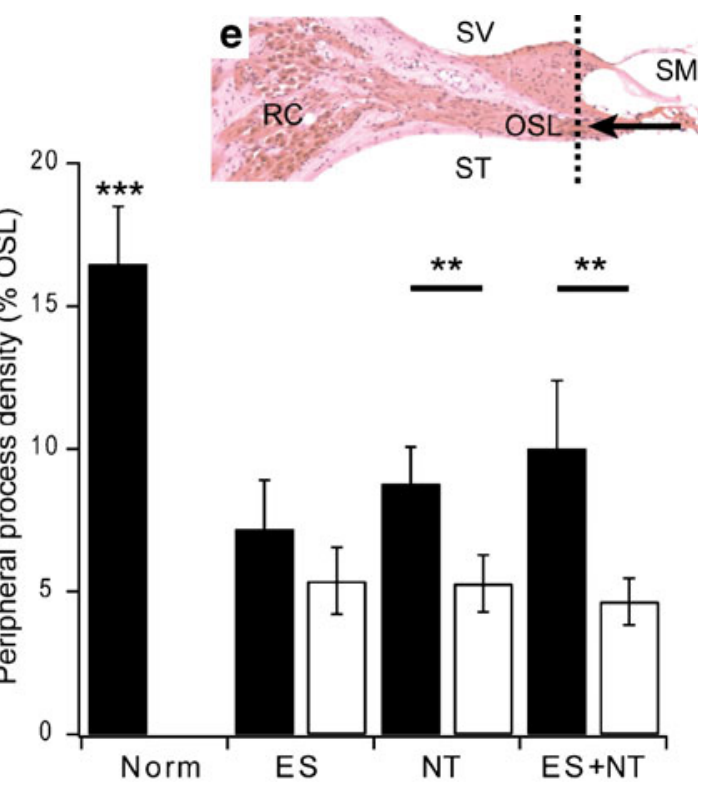

The mean $( \pm$ SEM) of peripheral process packing density expressed as a percentage of the OSL. The density of processes in NT and ES + NT treated cochleae was significantly greater compared to the deafened, untreated control cochleae. In contrast, there was no difference in peripheral process packing density in ES treated cochleae when compared to the deafened controls. (e) A histological micrograph depicting the region of the cochlear (dotted line) where the peripheral processes were measured within the OSL. (a-c) The arrow represents the cross-sectional view depicted. $\mathrm{RC}=$ Rosenthal's canal; $\mathrm{SM}=$ scala media; $\mathrm{SV}=$ scala vestibuli; $\mathrm{ST}=$ scala tympani. ${ }^{*}$ - significant difference

\section{Discussion}

This study has shown that cell-based delivery of NTs promoted the survival of SGNs and their peripheral processes following long-term deafness, and the extent of survival was enhanced when combined with cochlear implant use. Implantation of encapsulated NTCells was compatible with cochlear implant use in terms of surgical access, tissue biocompatibility, and chronic neural excitation, indicating that this therapy is both effective and clinically viable. A major advantage of the present study is the use of a long-term neonatal deafness model with chronic ES delivered by clinical devices, a model of early cochlear implantation for deaf infants.

\section{Effects of Chronic ES on SGN Survival}

There was no significant effect of chronic ES alone on SGN survival, a finding consistent with previous studies in the cat $[27,31,32]$ and guinea pig $[8,33,34]$, although this is not a uniform finding [25, 35-41]. Differences in the methodology (e.g., the duration and severity of deafness, the age at deafness onset, electrode design and position, duration, and mode of chronic ES) complicate direct 

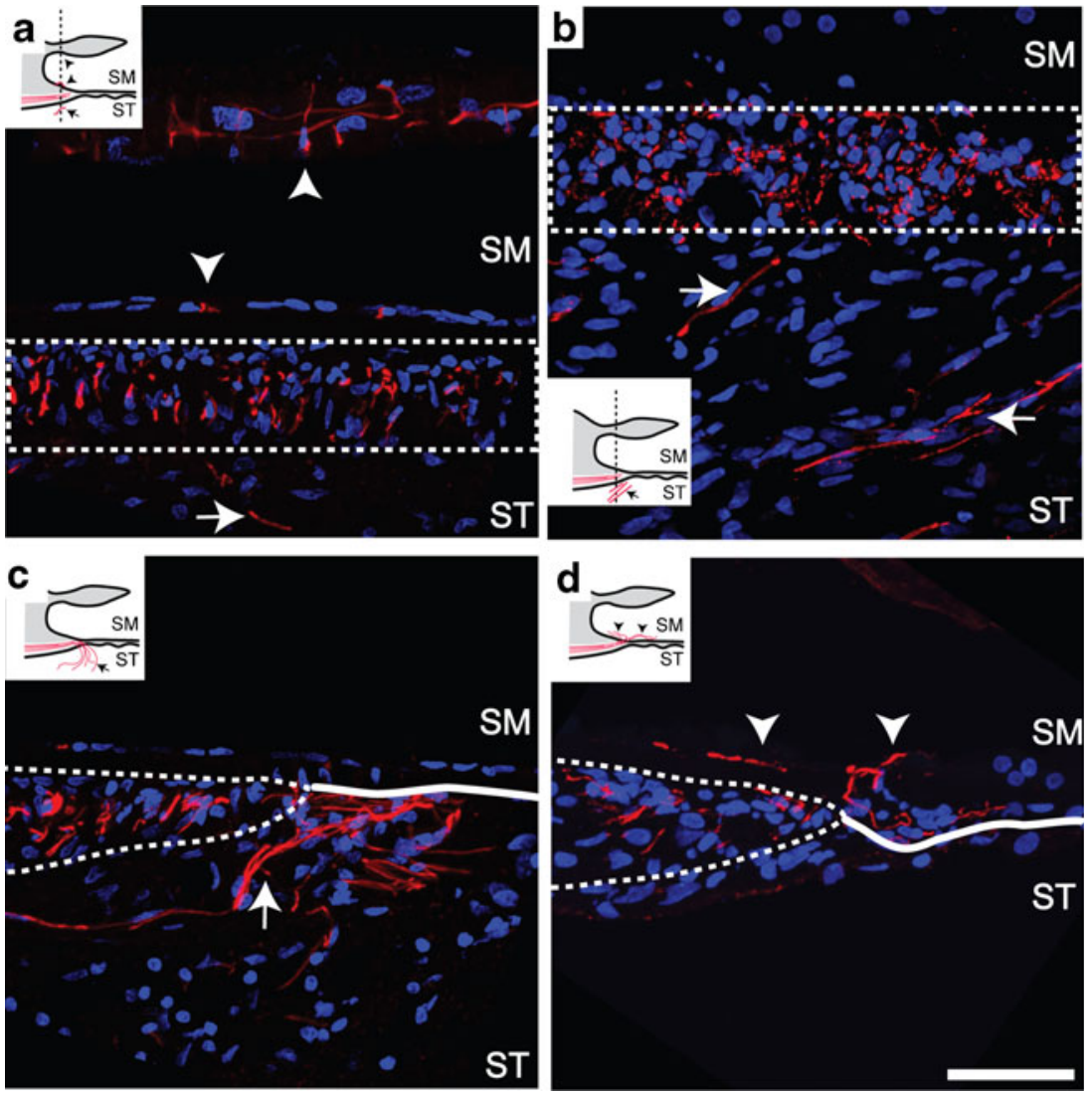

Fig. 7 Confocal images of cochlear sections stained with NF200 (red) and DAPI (blue). Dotted lines indicate the osseous spiral lamina (OSL) (a-d) and solid lines indicate the basilar membrane (c and d). Schematic inset diagrams indicate the sectioning plane and depict the process projections. (a and b) In OSL cross sections, re-sprouting processes were observed in the scala tympani (arrow) and within the cellular layer on the inner sulcus (downward arrowhead) or under the lip of the spiral limbus (upward arrowhead) in an ES-treated cochlea. Re-sprouting processes were also observed projecting laterally within

the scala tympani, running approximately parallel with the electrode array (not visible) within the cellular matrix generated by the tissue response to the electrode array (DAPI-stained cells). (c) In midmodiolar sections re-sprouting processes were observed to project into the scala tympani below the basilar membrane (solid line) in an EStreated cochlea. (d) Processes were also observed to project onto the basilar membrane and backwards within the inner sulcus (downward arrowhead) in an NT-treated cochlea. Scale bar $=50 \mu \mathrm{m}$. SM $=$ scala media; $\mathrm{ST}=$ scala tympani
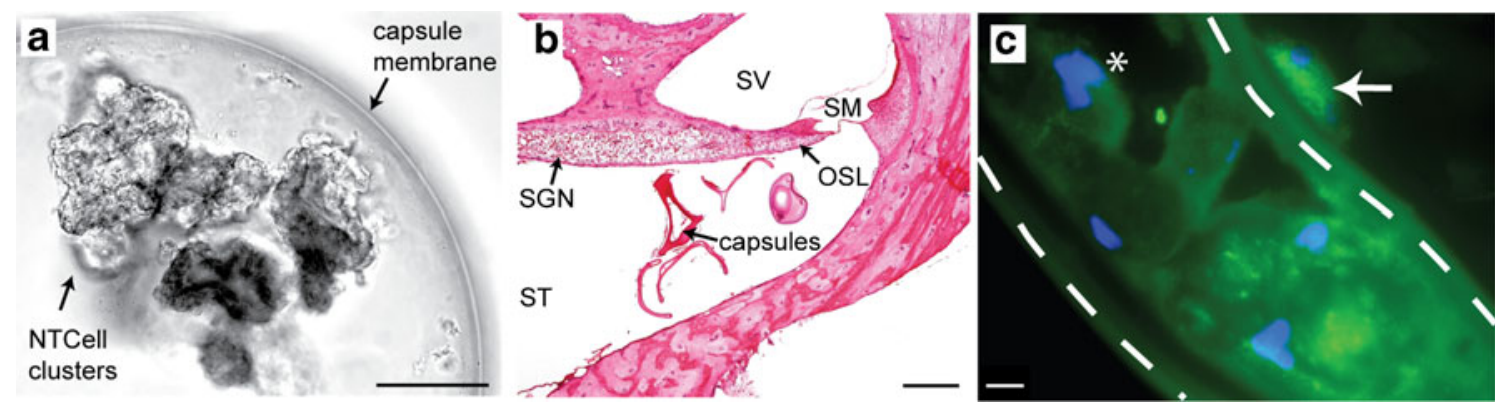

Fig. 8 a A capsule containing clusters of NTCells prior to implantation. The capsules were spherically shaped with a diameter of 600 to $660 \mu \mathrm{m}$ (scale bar $=100 \mu \mathrm{m}$ ). A number of clusters of NTCells are suspended inside the fluid-filled capsule. b The capsules were implanted into the scala tympani (ST) and were in close proximity to the spiral ganglion neurons (SGNs) and peripheral processes within the osseous spiral lamina (OSL) in the upper basal turn. Although the capsules were distorted during histological processing (arrow), there was minimal tissue response to the implanted capsules (scale bar $=500 \mu \mathrm{m})$. c An immunohistological image showing a cluster of NTCells within a capsule (white dotted line). There was positive staining for DAPI (*blue) and for mitochondria using cytochrome $\mathrm{C}$ oxidase (green), providing evidence that NTCells were alive and metabolically active at the conclusion of the treatment period. Note the presence of what is presumed to be a fibroblast on the outside of the capsule (arrow) indicative of a limited tissue response to the capsules (scale bar $=10 \mu \mathrm{m}) . \mathrm{SM}=$ scala media; $\mathrm{SV}=$ scala vestibuli 
Fig. 9 Example images of cochlear sections at location ii (a) and location iv (b) illustrates the extent of tissue response associated with cochlear implantation (see inset in Fig. 1b). New bone growth (a, arrow) in the lower basal turn and a capsule $(*)$ in the middle turn (a) and upper basal turn (b) are evident. There was minimal tissue response to the presence of the capsules. Scale bar $200 \mu \mathrm{m}$, Average ( \pm SEM) tissue response (c) and new bone growth (d) as a percentage of the scala tympani area. There was no statistical difference between the treatment conditions. ES = electrical stimulation; NT $=$ neurotrophins; RC = Rosenthal's canal; $\mathrm{SM}=$ scala media; $\mathrm{SV}=$ scala vestibuli; $\mathrm{ST}=$ scala tympani.

* - significant difference

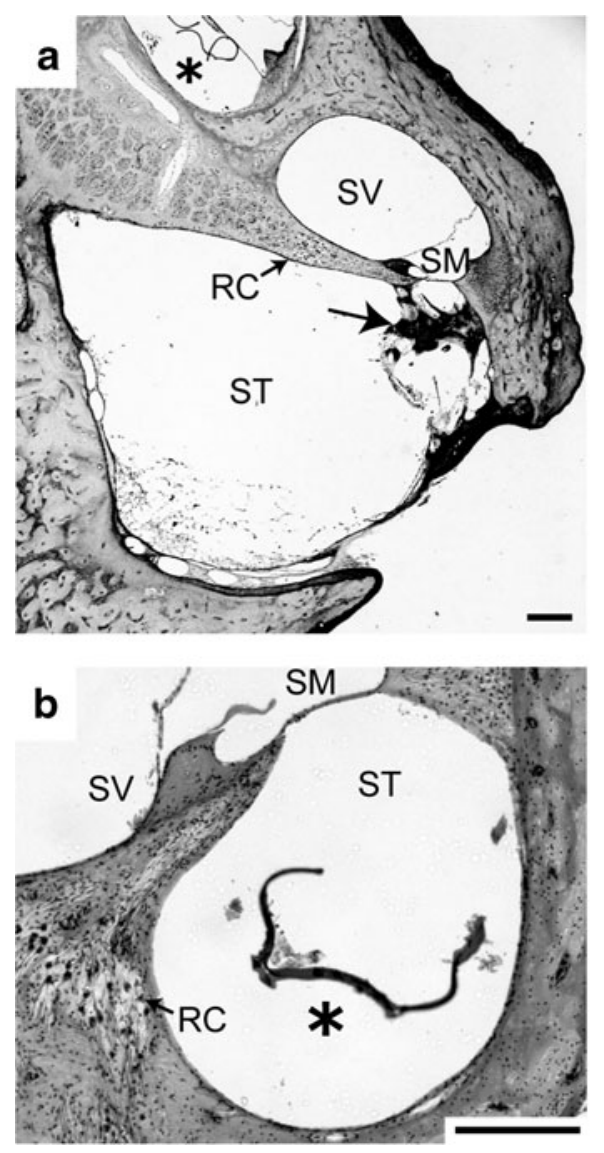

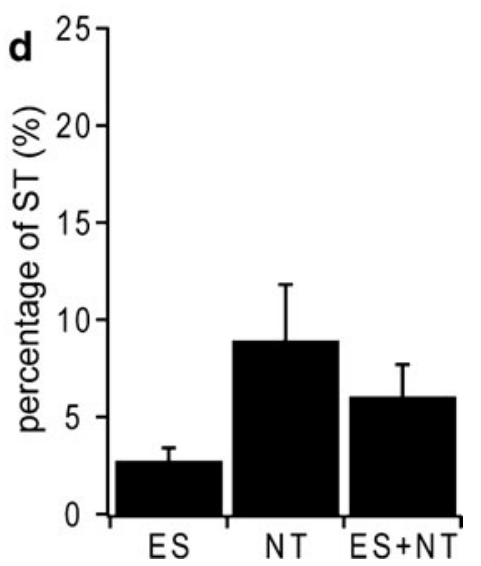

comparisons between these experiments [42]. However, a lack of ES-induced survival effects on SGNs reported here is supported by evidence from human temporal bones following cochlear implant use [43-47], although clinical studies lack the control offered by animal experiments.

\section{Effects of NT Treatment on SGN Survival}

The choroid plexus produces and secretes a wide range of NTs and other cell survival factors at physiological levels $(\mathrm{pg} / \mathrm{mL})[24,48]$. Significant SGN survival for the NT treatment group was observed in the upper basal cochlear region only ( $35 \%$ greater SGN density compared to the contralateral deafened untreated cochleae), providing evidence that the NTs released by the NTCells were protective for SGNs in this cochlear region. In addition to the production of NTs, the choroid plexus also produces antioxidants that can prevent oxidative stress in neurons by destroying oxygen free radicals in addition to limiting their production. The expression levels of anti-oxidants are in the top 5 to $10 \%$ of all genes expressed [24], and could represent another mechanism, whereby encapsulated NTCells exerted their protective effects on SGNs. Immunohistochemistry on the cat cochlea (Fig. 8) indicated that the NTCells were positively labeled with DAPI and cytochrome $\mathrm{C}$ oxidase, providing evidence that they were alive and metabolically active at the conclusion of a treatment period of at least 6 months. In a previous study, the viability of implanted capsules containing NTCells was assessed following implantation in the striatum of rats. After 4 months of implantation, NTCell viability was the same as pre-implant levels, but decreased to $67.5 \%$ following 6 months of implantation [24]. Therefore, it is likely that there was a reduction in NT production and release during the timeframe of the current experiments.

Capsules containing NTCells were observed primarily in the lower and upper basal cochlear regions (Figs. 5a and $8 \mathrm{~b}$ ), and infrequently in more apical cochlear regions (middle turn) (Fig. 9a). The region-specific survival effect on SGNs in the NT treatment group is likely to reflect local differences in the effective concentration of NTs available to the SGNs, and there are a number of factors that may contribute to the finding. Unlike the use of mini-osmotic pumps, whereby relatively high levels of NTs were delivered (for more detail see Ernfors et al. [9], Staecker et al. [10], Miller et al. [11], Wise [12], McGuinness and Shepherd [13], Glueckert et al. [14], and Agterberg et al. [15]), NTCells produce and release more physiological levels of NTs and therefore physical barriers (e.g., fibrous tissue, or simply the distance between the capsules and the 
target neurons) are far more critical. In addition, the relatively large area of the lower basal turn of the scala tympani $\left(\sim 5.0 \mathrm{~mm}^{2}\right)$ compared to the upper basal turn $\left(\sim 0.5 \mathrm{~mm}^{2}\right)$ [49] meant that the capsules containing NTCells were located relatively further away from the SGNs located within Rosenthal's canal, potentially reducing the local concentration of NTs in this region.

\section{Effects of Combined ES and NT Treatment on SGN Survival}

Combined ES + NT treatment resulted in significantly greater SGN density throughout a large extent of the cochlea (i.e., in the lower basal [44\%], upper basal [68\%], and lower middle [63\%] regions). Previous studies have shown that chronic ES can potentiate the pro-survival effects of growth factors such as NTs [8, 41]. ES may lead to an up-regulation of NT receptors [50-52] or increased intracellular trafficking of NT receptors that amplify the neuronal responsiveness to limited amounts of NTs in vivo [53]. ES also enhances the internalization of NT receptor complexes [54] (a process that is critical for neuronal responses to target-derived NTs [55]), and may play an important role in the enhanced SGN survival seen with the combined treatment. Another possibility is an ES-induced increase in the production and secretion of neuroprotective factors by the NTCells themselves. Finally, the regional specific effects of combined ES + NT treatment (no enhanced SGN survival in the upper middle turn) may also relate to the SGN population that was electrically activated throughout the treatment period. A previous study has shown enhanced SGN survival in cochlear regions likely to be electrically activated by chronic ES following long-term NT delivery [18].

\section{Peripheral Process Responses}

There was significantly greater density of peripheral processes within the OSL in cochleae that received NT treatment than in the untreated control cochleae, with the largest average density observed in the combined ES + NT treatment group. The increased process density is likely to be a combination of NT-induced protection of the peripheral processes and enhanced process re-sprouting and/or branching. Indeed, it was noticeable that the projection profiles of processes within the OSL of treated cochleae were different to the normal radial projection profile that typically exhibits punctate neurofilament staining in the process cross sections. The staining of processes in treated cochleae was less punctate, indicative of an increased ectopic projection of the processes within the OSL in these cochleae.

Previous studies have shown that NTs delivered to the deafened cochlea via a mini-osmotic pump can promote re- sprouting of the peripheral processes in the guinea pig cochlea $[12,14]$. In the present study, re-sprouting peripheral processes were observed in all treatment conditions, including cochleae that received ES only. Processes were observed within the scala media, projecting onto the basilar membrane and also coursing on the inner sulcus and up to the tectorial membrane. Importantly, re-sprouting processes were observed within the scala tympani of implanted cochleae and were incorporated into the fibrous tissue matrix associated with the tissue response to the intracochlear electrode within the scala tympani. Extensive re-sprouting, particularly if processes project longitudinally along the electrode array as reported in this study (Fig. 7b), may act to reduce the specificity of electrical stimulation delivered by a multi-channel cochlear implant, a factor that may contribute to the variability in implant performance observed between cochlear implant recipients; this is currently under investigation.

\section{Tissue Response to Treatment}

As with the implantation of any foreign body, there is an immune response from the cochlea to the presence of an electrode array. In the current study, cochlear implantation was associated with a loose fibrotic response, a tissue capsule surrounding the electrode array, and in some instances small areas of new bone growth that was typically restricted to the scala tympani of the basal turn. The extent of the tissue response and new bone growth to cochlear implantation was consistent to a previous report in which a similar electrode array (without capsules) was chronically implanted into the basal turn of the cat [56]. The presence of a tissue response in the present study was likely to contribute to the increase in electrode impedance that was observed during the treatment period. Importantly, there was no significant difference in the tissue response for cochleae that were implanted with empty capsules and those that received NTCells, indicating that the factors released did not promote osteoneogenesis or cause significant increases in the tissue response. Indeed, capsules were often associated with very few inflammatory cells (Figs. 5, 8, and 9), indicating that the capsule membrane provided a robust immunological barrier to the porcine NTCells. Therefore, the role of the choroid plexus in the production of NTs and other factors (at physiologically relevant levels) makes it an attractive option for SGN protection that is safe and compatible with the cochlear environment.

One methodological issue warrants discussion. Higher SGN densities were observed in the contralateral (unimplanted) cochleae of the ES group compared to the NT and ES + NT group. A factor likely contributing to this result is the shorter duration of deafness in the ES group (Table 1). All statistical analysis used "within 
animal" comparisons, taking advantage of the symmetrical degeneration following systemic neomycin administration [5], and therefore eliminating the influence of any differences in neural survival between animals.

\section{Clinical Implications and Future Directions}

It is commonly believed that a robust population of SGNs is beneficial for cochlear implant performance. However, to date it has not been possible to show a positive correlation between cochlear implant performance and SGN density $[45,46,57$, 58]. The conductive nature of the cochlear fluids leads to relatively low spatial precision of SGN activation by cochlear ES meaning that higher SGN densities may not offer improvements in resolution with contemporary devices. New electrode designs that can benefit from biological interventions aimed at protecting the SGNs and regenerating their peripheral processes coupled with new stimulation strategies aimed at delivering more focused ES are likely to lead to improvements in cochlear implant performance in the future [59, 60]. Moreover, the development of high resolution electrode arrays for direct implantation into the auditory nerve [61] would rely on very good neural survival. Finally, future interventions that combine drug delivery and new electrode designs may be able to take advantage of the re-sprouting capacity of SGN peripheral processes to achieve a more direct interface between the electrode and the nerve to provide high resolution electrical stimulation.

This study has shown that cell-based delivery of therapeutic agents can be effectively combined with a cochlear implant, and therefore it is likely to be a viable strategy of protecting neural populations when combined with other neural prostheses, such as deep brain or retinal stimulation. Neuroprotective agents produced and released via cell-based techniques may reduce or prevent changes at the nerve-electrode interface, such as cell loss and scar tissue formation, which can reduce the efficacy of therapeutic electrical stimulation.

Acknowledgments The authors would like to acknowledge the contributions of Jin Xu, Helen Feng, Maria Clarke, Prudence Nielsen, Anette Fransson, Mohannad Fallatah and Jacqueline Andrew. Funding was provided by the National Institutes of Health (HHS-N-263-200700053-C), The Garnett Passe and Rodney Williams Memorial Foundation, and the National Health and Medical Research Council. The authors would like to acknowledge the support from the State Government of Victoria's Operational Infrastructure Program.

A full conflict of interest disclosure is available in the electronic supplementary material for this article.

\section{References}

1. Spoendlin H. Retrograde degeneration of the cochlear nerve. Acta Otolaryngol 1975;79:266-275.
2. Webster M, Webster DB. Spiral ganglion neuron loss following organ of Corti loss: a quantitative study. Brain Res 1981;212:1730.

3. Hardie NA, Shepherd RK. Sensorineural hearing loss during development: morphological and physiological response of the cochlea and auditory brainstem. Hear Res 1999;128:147-165.

4. Leake PA, Hradek GT. Cochlear pathology of long term neomycin induced deafness in cats. Hear Res 1988;33:11-33.

5. Xu SA, Shepherd RK, Chen Y, Clark GM. Profound hearing loss in the cat following the single co-administration of kanamycin and ethacrynic acid. Hear Res 1993;70:205-215.

6. Ernfors P, Merlio JP, Persson H. Cells Expressing mRNA for Neurotrophins and their Receptors During Embryonic Rat Development. Eur J Neurosci 1992;4:1140-1158.

7. Ylikoski J, Pirvola U, Moshnyakov M, Palgi J, Arumae U, Saarma M. Expression patterns of neurotrophin and their receptor mRNAs in the rat inner ear. Hear Res 1993;65:69-78.

8. Shepherd RK, Coco A, Epp SB, Crook JM. Chronic depolarization enhances the trophic effects of brain-derived neurotrophic factor in rescuing auditory neurons following a sensorineural hearing loss. J Comp Neurol 2005;486:145-158.

9. Ernfors P, Duan ML, ElShamy WM, Canlon B. Protection of auditory neurons from aminoglycoside toxicity by neurotrophin-3. Nat Med 1996;2:463-467.

10. Staecker H, Kopke R, Malgrange B, Lefebvre P, Van de Water TR. NT-3 and/or BDNF therapy prevents loss of auditory neurons following loss of hair cells. Neuroreport 1996;7:889-894.

11. Miller JM, Chi DH, O'Keeffe LJ, Kruszka P, Raphael Y, Altschuler RA. Neurotrophins can enhance spiral ganglion cell survival after inner hair cell loss. Int J Dev Neurosci 1997;15:631-643.

12. Wise AK, Richardson R, Hardman J, Clark G, O'Leary S. Resprouting and survival of guinea pig cochlear neurons in response to the administration of the neurotrophins brain-derived neurotrophic factor and neurotrophin-3. J Comp Neurol 2005;487:147-165.

13. McGuinness SL, Shepherd RK. Exogenous BDNF rescues rat spiral ganglion neurons in vivo. Otol Neurotol 2005;26:10641072.

14. Glueckert R, Bitsche M, Miller JM, et al. Deafferentationassociated changes in afferent and efferent processes in the guinea pig cochlea and afferent regeneration with chronic intrascalar brain-derived neurotrophic factor and acidic fibroblast growth factor. J Comp Neurol 2008;507:1602-1621.

15. Agterberg MJ, Versnel H, De Groot JC, Smoorenburg GF, Albers FW, Klis SF. Morphological changes in spiral ganglion cells after intracochlear application of brain-derived neurotrophic factor in deafened guinea pigs. Hear Res 2008;244:25-34.

16. Landry TG, Wise AK, Fallon JB, Shepherd RK. Effects of combined intracochlear neurotrophin infusion and chronic electrical stimulation on spiral ganglion neuron survival and function. Hear Res 2011;(in press).

17. Gillespie LN, Clark GM, Bartlett PF, Marzella PL. BDNF-induced survival of auditory neurons in vivo: Cessation of treatment leads to accelerated loss of survival effects. J Neurosci Res 2003;71:785790 .

18. Shepherd RK, Coco A, Epp SB. Neurotrophins and electrical stimulation for protection and repair of spiral ganglion neurons following sensorineural hearing loss. Hear Res 2008;242:100109.

19. Nadol JB, Jr., Young YS, Glynn RJ. Survival of spiral ganglion cells in profound sensorineural hearing loss: implications for cochlear implantation. Ann Otol Rhinol Laryngol 1989;98:411416.

20. Gillespie LN, Shepherd RK. Clinical application of neurotrophic factors: the potential for primary auditory neuron protection. Eur J Neurosci 2005;22:2123-2133. 
21. Apfel SC. Is the therapeutic application of neurotrophic factors dead? Ann Neurol 2002;51:8-11.

22. Emerich DF, Vasconcellos AV, Elliott RB, Skinner SJ, Borlongan $\mathrm{CV}$. The choroid plexus: function, pathology and therapeutic potential of its transplantation. Expert Opin Biol Ther 2004;4:1191-1201.

23. Pettingill LN, Wise AK, Geaney MS, Shepherd RK. Enhanced auditory neuron survival following cell-based BDNF treatment in the deaf guinea pig. PLoS One 2011;6:e18733.

24. Skinner SJ, Geaney MS, Lin H, et al. Encapsulated living choroid plexus cells: potential long-term treatments for central nervous system disease and trauma. J Neural Eng 2009;6:065001.

25. Leake PA, Hradek GT, Rebscher SJ, Snyder RL. Chronic intracochlear electrical stimulation induces selective survival of spiral ganglion neurons in neonatally deafened cats. Hear Res 1991;54:251-271.

26. Fallon JB, Irvine DR, Shepherd RK. Cochlear implant use following neonatal deafness influences the cochleotopic organization of the primary auditory cortex in cats. J Comp Neurol 2009;512:101-114.

27. Coco A, Epp SB, Fallon JB, Xu J, Millard RE, Shepherd RK. Does cochlear implantation and electrical stimulation affect residual hair cells and spiral ganglion neurons? Hear Res 2007;225:60-70.

28. Borlongan CV, Skinner SJ, Geaney M, Vasconcellos AV, Elliott $\mathrm{RB}$, Emerich DF. Intracerebral transplantation of porcine choroid plexus provides structural and functional neuroprotection in a rodent model of stroke. Stroke 2004;35:22062210.

29. Emerich DF, Lindner MD, Winn SR, Chen EY, Frydel BR, Kordower JH. Implants of encapsulated human CNTF-producing fibroblasts prevent behavioral deficits and striatal degeneration in a rodent model of Huntington's disease. J Neurosci 1996;16:51685181.

30. Wise AK, Hume CR, Flynn BO, et al. Effects of localized neurotrophin gene expression on spiral ganglion neuron resprouting in the deafened cochlea. Mol Ther 2010;18:1111-1122.

31. Araki S, Kawano A, Seldon L, Shepherd RK, Funasaka S, Clark GM. Effects of chronic electrical stimulation on spiral ganglion neuron survival and size in deafened kittens. Laryngoscope 1998;108:687-695.

32. Shepherd RK, Matsushima J, Martin RL, Clark GM. Cochlear pathology following chronic electrical stimulation of the auditory nerve: II. Deafened kittens. Hear Res 1994;81:150-166.

33. Li L, Parkins CW, Webster DB. Does electrical stimulation of deaf cochleae prevent spiral ganglion degeneration? Hear Res 1999; 133:27-39.

34. Agterberg MJ, Versnel H, de Groot JC, van den Broek M, Klis SF. Chronic electrical stimulation does not prevent spiral ganglion cell degeneration in deafened guinea pigs. Hear Res 2010;269:169179.

35. Leake PA, Snyder RL, Merzenich MM. Topographic organization of the cochlear spiral ganglion demonstrated by restricted lesions of the anteroventral cochlear nucleus. J Comp Neurol 1992;320:468-478.

36. Snyder R, Leake P, Rebscher S, Beitel R. Temporal resolution of neurons in cat inferior colliculus to intracochlear electrical stimulation: effects of neonatal deafening and chronic stimulation. J Neurophysiol 1995;73:449-467.

37. Leake PA, Hradek GT, Snyder RL. Chronic electrical stimulation by a cochlear implant promotes survival of spiral ganglion neurons after neonatal deafness. J Comp Neurol 1999;412:543562.

38. Lousteau RJ. Increased spiral ganglion cell survival in electrically stimulated, deafened guinea pig cochleae. Laryngoscope 1987;97 (7pt 1):836-842.
39. Hartshorn DO, Miller JM, Altschuler RA. Protective effect of electrical stimulation in the deafened guinea pig cochlea. Otolaryngol Head Neck Surg 1991;104:311-319.

40. Mitchell A, Miller JM, Finger PA, Heller JW, Raphael Y, Altschuler RA. Effects of chronic high-rate electrical stimulation on the cochlea and eighth nerve in the deafened guinea pig. Hear Res 1997;105:30-43.

41. Kanzaki S, Stover T, Kawamoto K, et al. Glial cell line-derived neurotrophic factor and chronic electrical stimulation prevent VIII cranial nerve degeneration following denervation. J Comp Neurol 2002;454:350-360.

42. Shepherd RK, Meltzer NE, Fallon JB, Ryugo DK. Consequences of deafness and electrical stimulation on the peripheral and central auditory system. In: Waltzman SB, Roland TJ, eds. Cochlear Implants, 2nd ed. New York: Thieme Medical Publishers, Inc., 2006:25-39.

43. Linthicum FH Jr, Fayad J, Otto SR, Galey FR, House WF. Cochlear implant histopathology. Am J Otol 1991;12:245-311.

44. Nadol JB Jr, Ketten DR, Burgess BJ. Otopathology in a case of multichannel cochlear implantation. Laryngoscope 1994;104(3pt 1):299-303.

45. Nadol JB Jr, Shiao JY, Burgess BJ, et al. Histopathology of cochlear implants in humans. Ann Otol Rhinol Laryngol 2001;110:883-891.

46. Khan AM, Handzel O, Damian D, Eddington DK, Nadol JB Jr. Effect of cochlear implantation on residual spiral ganglion cell count as determined by comparison with the contralateral nonimplanted inner ear in humans. Ann Otol Rhinol Laryngol 2005;114:381-385.

47. Nadol JB Jr, Eddington DK. Histopathology of the inner ear relevant to cochlear implantation. Adv Otorhinolaryngol 2006;64:31-49.

48. Johanson CE, Palm DE, Primiano MJ, et al. Choroid plexus recovery after transient forebrain ischemia: role of growth factors and other repair mechanisms. Cell Mol Neurobiol 2000;20:197216.

49. Hatsushika S, Shepherd RK, Tong YC, Clark GM, Funasaka S. Dimensions of the scala tympani in the human and cat with reference to cochlear implants. Ann Otol Rhinol Laryngol 1990;99:871-876.

50. Meyer-Franke A, Wilkinson GA, Kruttgen A, et al. Depolarization and cAMP elevation rapidly recruit TrkB to the plasma membrane of CNS neurons. Neuron 1998;21:681-693.

51. Du J, Feng L, Yang F, Lu B. Activity- and $\mathrm{Ca}(2+)$-dependent modulation of surface expression of brain-derived neurotrophic factor receptors in hippocampal neurons. J Cell Biol 2000;150:1423-1434.

52. Zhao L, Sheng AL, Huang SH, et al. Mechanism underlying activity-dependent insertion of TrkB into the neuronal surface. $\mathrm{J}$ Cell Sci 2009;122(pt 17):3123-3136.

53. Ascano M, Richmond A, Borden P, Kuruvilla R. Axonal targeting of Trk receptors via transcytosis regulates sensitivity to neurotrophin responses. J Neurosci 2009;29:1167411685.

54. Du J, Feng L, Zaitsev E, Je HS, Liu XW, Lu B. Regulation of TrkB receptor tyrosine kinase and its internalization by neuronal activity and Ca2+ influx. J Cell Biol 2003;163: 385-395.

55. Zhang Y, Moheban DB, Conway BR, Bhattacharyya A, Segal RA. Cell surface Trk receptors mediate NGF-induced survival while internalized receptors regulate NGF-induced differentiation. J Neurosci 2000;20:5671-5678.

56. Xu J, Shepherd RK, Millard RE, Clark GM. Chronic electrical stimulation of the auditory nerve at high stimulus rates: a physiological and histopathological study. Hear Res 1997;105:129. 
57. Blamey P. Are spiral ganglion cell numbers important for speech perception with a cochlear implant? Am J Otol 1997;18(6 suppl): S11-S12.

58. Khan AM, Handzel O, Burgess BJ, Damian D, Eddington DK, Nadol JB Jr. Is word recognition correlated with the number of surviving spiral ganglion cells and electrode insertion depth in human subjects with cochlear implants? Laryngoscope 2005;115:672-677.
59. van den Honert C, Kelsall DC. Focused intracochlear electric stimulation with phased array channels. J Acoust Soc Am 2007;121:3703-3716.

60. Bierer JA. Probing the electrode-neuron interface with focused cochlear implant stimulation. Trends Amplif 2010;14:84-95.

61. Middlebrooks JC, Snyder RL. Intraneural stimulation for auditory prosthesis: modiolar trunk and intracranial stimulation sites. Hear Res 2008;242:52-63. 